\title{
ON GENERALIZATIONS OF LENGTH AND AREA $†$
}

\author{
BY J. F. RANDOLPH
}

1. Introduction. In the Lebesgue theory of integration, if $G$ is a bounded Lebesgue measurable point set on the $x$ axis and $f(x)$ is a non-negative bounded Lebesgue measurable function on $G$, then the plane set $H$, consisting of all points $(x, y)$ such that $x$ is on $G$ and $0 \leqq y \leqq f(x)$, is Lebesgue plane measurable and $m^{(2)} H=\int_{G} f(x) d x$. This relation may be proved by showing first that if $h$ is a positive number, then the set $H_{h}:[x$ in $G, 0 \leqq y \leqq h]$ is Lebesgue plane measurable and

$$
m^{(2)} H_{h}=h m G,
$$

that is, the "area" of $H_{h}$ is its "base" times its "altitude".

C. Carathéodory, W. Gross, and others have defined linear measure for sets not necessarily on a line, plane measure for sets not necessarily on a plane, and in general p-dimensional measure for sets in $q$-dimensional space, which are generalizations of the notion of curve length, surface area, and so on. Fundamental and simple as it seems, the question whether the generalizations of length and area under these definitions preserve, as do Lebesgue's, the euclidean relation that area is the product of length by length, has received no attention. In this paper we discuss, without answering completely, the simplest phase of this question.

2. Axioms on General Measure. We first point out some facts concerning general measure.

By postulating the existence of a set function satisfying five axioms, Carathéodory [2] developed a general theory of measure in which most of the theorems of the usual Lebesgue theory have an analog. Hahn [4], page 444, modified Carathéodory's fifth axiom, and by its use proved also the important relation that the inner measure of a set is the upper limit of the measures of its closed subsets. Hahn's modified axiom is as follows.

$\dagger$ Presented to the Society, September 12, 1935.

$\ddagger$ Numbers in brackets refer to the bibliography. 
Axiom $V^{\prime}$. The outer measure of a set is the lower limit of the measures of all containing sets each of which is the intersection of a countable number of open sets.

3. Carathéodory's Specific Measures. Carathéodory also gave the following specific method of attaching a number $L^{*} A$ to a set $A$ in a euclidean space $R_{q}$ of $q$ dimensions.

With $\rho$ a positive number let $U_{1}, U_{2}, \cdots$ be a sequence of convex point sets open in the space $R_{q}, \dagger$ each with diameter less than $\rho$, whose union contains $A$. With $d_{k}$ the diameter of $U_{k}$ consider the sums $d_{1}+d_{2}+\cdots$ for all such sequences of point sets. Designate the greatest lower bound, which may be $+\infty$, of such sums by $L_{\rho} A$. Then $L_{\rho} A$ does not decrease as $\rho$ decreases. Thus as $\rho \rightarrow 0, L_{\rho} A$ approaches a limit, finite or infinite, which in either case is called the outer linear measure of $A$ and is represented by $L^{*} A$.

The outer plane measure $L^{*(2)} A$ of a set $A$ is also defined by means of open convex sets $U_{1}, U_{2}, \cdots$, each with diameter less than $\rho$, except that $d_{k}$ is replaced by the two-dimensional diameter of $U_{k}$. The two-dimensional diameter of an open convex set is the least upper bound of the Lebesgue plane measures of the projections of the set on planes of all possible orientations. Arguments similar to those for linear measure show that plane measure also satisfies Hahn's axiom.

4. Statement of the Question. The simplest phase of the question under discussion for Carathéodory measure may now be stated as follows.

If $A$ is a plane Carathéodory linearly measurable point set with $L A$ finite, is the cylindrical set $J_{h}:[(x, y)$ in $A, 0 \leqq z \leqq h]$ Carathêodory plane measurable and if so is $L^{(2)} J_{h}=h L A$ ?

5. An Inequality for Carathéodory's Measures. We first prove the following theorem.

Theorem 1. If the set $A$ has outer linear measure $L^{*} A$ finite, then the outer plane measure $L^{*(2)} J_{h}$ of $J_{h}$ is less than or equal to $h L^{*} A$.

With $\rho$ a positive number less than unity, let $U_{1}, U_{2}, \cdots$

$\dagger$ Carathéodory did not assume the sets $U_{k}$ to be open, but proved that the same number would be obtained if open convex sets were used. 
be a sequence of plane convex point sets whose union contains $A$ and for which $d_{k}<\rho^{2} / 2$ and $\sum_{k} d_{k}<L^{*} A+\rho$. Now let $N_{k}$ be the sequence of integers such that $h \leqq N_{k}\left(d_{k}\right)^{1 / 2} \leqq h+\left(d_{k}\right)^{1 / 2}$ and form the three dimensional sets $V_{k n}$ of all points $(x, y, z)$ such that $(x, y)$ is in $U_{k}$ and $(n-1)\left(d_{k}\right)^{1 / 2} \leqq z \leqq n\left(d_{k}\right)^{1 / 2},\left(n=1,2, \cdots, N_{k}\right)$.

Then each $V_{k n}$ may be included in a right circular cylinder with diameter of base $d_{k}$ and altitude $\left(d_{k}\right)^{1 / 2}$. Consequently the diameter of $V_{k n}$ is not greater than $\left(d_{k}^{2}+d_{k}\right)^{1 / 2}<\rho$. Thus, with $D_{k n}$ the two-dimensional diameter of $V_{k n}$, we have

$$
L_{\rho}{ }^{(2)}\left(J_{h}\right) \leqq \sum_{k} \sum_{n} D_{k n} .
$$

But the projection on any plane of a right circular cylinder with diameter of base $\delta$ and altitude $\alpha$ may be included in a rectangle with dimensions $\delta$ and $\left(\delta^{2}+\alpha^{2}\right)^{1 / 2}$. Thus $D_{k n} \leqq d_{k}\left(d_{k}^{2}+d_{k}\right)^{1 / 2}$ $=d_{k}\left(d_{k}\right)^{1 / 2}\left(d_{k}+1\right)^{1 / 2}<\left(d_{k}\right)^{1 / 2}(\rho+1)^{1 / 2} d_{k}$. So $\quad \sum_{n} D_{k n} \leqq N_{k}\left(d_{k}\right)^{1 / 2}$ $\cdot(\rho+1)^{1 / 2} d_{k} \leqq\left(h+\left(d_{k}\right)^{1 / 2}\right)(\rho+1)^{1 / 2} d_{k}<(h+\rho)(\rho+1)^{1 / 2} d_{k}$. Consequently $L_{\rho}{ }^{(2)}\left(J_{h}\right) \leqq(h+\rho)(\rho+1)^{1 / 2} \sum_{k} d_{k}<(h+\rho)(\rho+1)^{1 / 2}\left(L^{*} A+\rho\right)$. But this is true for each $\rho$ so $L^{*(2)} J_{h} \leqq h L^{*} A$. In particular, if $A$ is Carathéodory linearly measurable, then $J_{h}$ is plane measurable, and

$$
L^{(2)} J_{h} \leqq h L A .
$$

This follows since any cylindrical set of altitude $h$ with a closed subset $K$ of $A$ as base is a closed subset of $J_{h}$. Consequently, the outer plane measures of the part of $J_{h}$ not in this closed subset is less than or equal to $h L(A-K)$, which may be made arbitrarily small by a proper choice of $K$.

The question turns then on the reverse inequality

$$
L^{(2)} J_{h} \geqq h L A .
$$

This inequality, which seems on first thought the more plausible of the two, yields to no method of attack we have been able to devise.

6. Discussion of Two Measures. By introducing modifications $\Lambda A$ and $\Lambda^{(2)} J_{h}$ of Gross' definitions of measures, we establish an inequality corresponding to (b). The situation is, however, again disconcerting as we are unable to establish for these measures the inequality corresponding to (a). 
A comparison of the numbers assigned by the two definitions gives the desired relation between the generalizations of length and area for a certain class of sets. For, as we shall show, $L^{(2)} J_{h} \geqq \Lambda^{(2)} J_{h}$ and thus $h L A \geqq L^{(2)} J_{h} \geqq \Lambda^{(2)} J_{h} \geqq h \Lambda A$. Consequently, if $L A=\Lambda A$ then $h L A=L^{(2)} J_{h}=\Lambda^{(2)} J_{h}=h \Lambda A$. We also obtain conditions on the set $A$ sufficient for the equality $L A=\Lambda A$. Finally we show that our conditions are not necessary for the equality $h L A=L^{(2)} J_{h}$.

7. The Gross Outer Measure. Following Gross, [3], page 156, we fix a rectangular coordinate system and a positive integer $k$ and designate as a partial square the set of all points $(x, y)$ such that $p / 2^{k} \leqq x<(p+1) / 2^{k}, q / 2^{k} \leqq y<(q+1) / 2^{k}$, where $p$ and $q$ are integers. There is a countable number of partial squares containing the plane set $A$ which we designate by $w_{k}^{1}, w_{k}^{2}, \cdots$. Let $\delta_{k}{ }^{i}$ be the least upper bound of the Lebesgue outer measures of the projections of $A w_{k}^{i}$ on lines of the plane, and form the sums $S_{k}(A)=\sum_{i} \delta_{k}{ }_{k}^{i}$, which may be $+\infty$. Since $w_{k}^{i}$ consists of four partial squares $w_{k+1}^{i_{1}}, \cdots, w_{k+1}^{i_{4}}$, we have $\delta_{k}^{i} \leqq \delta_{k+1}^{i_{1}}+\cdots+\delta_{k+1}^{i_{4}}$. Consequently, $S_{k}(A) \leqq S_{k+1}(A)$, so that $\lim _{k \rightarrow \infty} S_{k}(A)$, which may be $+\infty$, exists. This limit is the Gross outer linear measure of $A$ and is represented by $\Phi_{0}{ }^{*}(A)$.

The Gross outer plane measure $\Phi_{0}^{*(2)}(A)$ of a three-dimensional set $A$ is defined in a similar manner.

This outer linear measure is shown by Gross to satisfy Carathéodory's first four axioms. Furthermore, it is seen that $\Phi_{0}{ }^{*}(A)$ is not less than the Lebesgue outer measure of the projection of $A$ on any line. Gross [3], page 158, shows that if $\bar{\Phi}_{0}^{*}(A)$ is any other set function satisfying the same four axioms and the projection relation, then $\Phi_{0}^{*}(A) \leqq \bar{\Phi}_{0}^{*}(A)$; that is, $\Phi_{0}^{*}(A)$ is the minimum among such measures and is consequently independent of the coordinate system.

8. The Outer $\Lambda$ Measures. The outer linear measure $\Lambda^{*} A$ we shall use is the greatest lower bound of the Gross linear measures of all those sets containing $A$ which are the intersections of sequences of open sets. Thus $\Lambda$ measure satisfies Carathéodory's first four axioms, Hahn's Axiom V', and the projection relation. Consequently, it follows that the inner $\Lambda$ measure $\Lambda_{*} A$ is the upper limit of the $\Lambda$ linear measures of closed subsets of $A$.

Furthermore, because Gross' measure is the "smallest" in 
the sense used above, $\Lambda$ measure is the minimum of the measures satisfying Axioms $\mathrm{I}_{-} \mathrm{V}^{\prime}$ and the projection relation. In particular, if $K$ is a closed subset of $A, \Lambda^{*}(A-K) \leqq L^{*}(A-K)$. We thus see that if $A$ is Carathéodory linearly measurable with $L A$ finite, then $A$ is also $\Lambda$ linearly measurable.

The converse of this relation-that if $A$ is $\Lambda$ linearly measurable, then $A$ is Carathéodory linearly measurable-does not follow. For Besicovitch [1], page 456, and Saks [6] have both constructed closed sets, with positive finite Carathéodory linear measure, whose projection on any line has Lebesgue measure zero. Consequently, any Carathéodory non-linearly measurable subset of either of these sets (if such a subset exists) is still $\Lambda$ linearly measurable since it has outer $\Lambda$ linear measure zero.

9. An Inequality for $\Lambda$ Measures. We shall now prove the following theorem.

Theorem 2. If $A$ is a plane $\Lambda$ linearly measurable point set with $\Lambda A$ finite then $\Lambda^{*(2)} J_{h} \leqq h \Lambda A . \dagger$

Let $w_{k}^{1}, w_{k}^{2}, \cdots$ be a sequence of partial squares whose union contains $A$. Then, with $W_{k}^{i j}$ the partial cube of all points $(x, y, z)$ such that $(x, y)$ is in $w_{k}^{i}$ and $j / 2^{k} \leqq z<(j+1) / 2^{k}$, we see that $\sum_{i} \sum_{j} W_{k}{ }^{i j}$ contains $J_{h}$. Then the projection $p^{i}$ of $A w_{k}^{i}$ on a line of the plane is Lebesgue measurable. For a closed subset $K$ of $A w_{k}^{i}$ projects into a closed subset $K_{p}$ of $p^{i}$ and $m^{*}\left(p^{i}-K_{p}\right) \leqq \Lambda\left(A w_{l}^{i}-K\right)$ and, from the $\Lambda$ measurability of $A w_{k}^{i}$, the right side of this inequality may be made arbitrarily small by a proper choice of $K$. Thus the set $P^{i j}$ of all points $(x, y, z)$ such that $(x, y)$ is in $p^{i}$ and $j / 2^{k} \leqq z<(j+1) / 2^{k}$ is a Lebesgue plane measurable set which is the projection of $J_{h} W_{k}^{i j}$ on a plane parallel to the $z$ axis. Consequently (see equality (A), \$1) $m^{(2)} P^{i j}=\left(1 / 2^{k}\right) m p^{i}$. Thus with $\Delta_{k}{ }^{i j}$ the least upper bound of the outer Lebesgue plane measures of the projections of $J_{h} W_{k}{ }^{i j}$ on planes of all possible orientations, $\Delta_{k}^{i j} \geqq\left(1 / 2^{k}\right) \delta_{k}^{i}$, and we see that $\Lambda^{*(2)} J_{h} \geqq h \Lambda A$.

10. A Sufficient Condition. We now see that a sufficient condition that $J_{h}$ be Carathéodory plane measurable and have

$\dagger$ The asterisk is used on the left to indicate outer measure since we are not able to prove here, as in the case of Carathéodory measure, that the measurability of $J_{h}$ follows from that of $A$. 
$L^{(2)} J_{h}=h L A$ is that $A$ be Carathéodory linearly measurable with $L A=\Lambda A$. Toward giving a condition under which $L A$ $=\Lambda A$ we make use of the density functions, as defined by Besicovitch [1], page 423. Following him, we shall call a point set $A$ regular if $A$ is Carathéodory linearly measurable and the density of $A$ exists and equals unity at almost all points of $A$.

From a special case of one of Besicovitch's theorems [1], page 426 , it follows that at almost all points $p$ of a regular set $A$ there exists a line $l(p)$ through $p$ such that $2 r$ and the Lebesgue measure of the projection $[A c(p, r)]_{l(p)}$ of $A c(p, r)$ on $l(p)$ are such that

$$
\lim _{r \rightarrow 0} \frac{m[A c(p, r)]_{l(p)}}{2 r}=1 .
$$

Since $m[A c(p, r)]_{l(p)} \leqq \Lambda A c(p, r) \leqq L A c(p, r)$, we then see that $\lim _{r \rightarrow 0} \Lambda A c(p, r) /(2 r)$ exists and also equals unity at all points $p$ of $A$ except a set of Carathéodory (and consequently $\Lambda$ ) linear measure zero.

Thus, corresponding to an arbitrary positive number $\epsilon$, there exists, for almost all points $p$ of $A$, a sequence of circles with radii approaching zero, for each circle $c(p, r)$ of which

$$
(1-\epsilon) 2 r<\Lambda A c(p, r) \leqq L A c(p, r)<(1+\epsilon) 2 r .
$$

Let $F$ represent the collection of all such circles. Since the density of $A$ is bounded from zero by unity for almost all of $A$, we see from [5] Lemma $1, \S 10$, that there exists in the family of circles $F$ a sequence of mutually exclusive circles $c(p, r)$ whose union contains almost all of $A$. We then observe that

$$
\begin{aligned}
L A & =\sum_{i} L A c\left(p_{i}, r_{i}\right)<(1+\epsilon) \sum_{i} 2 r_{i} \\
& <\frac{(1+\epsilon) \sum_{i} \Lambda A c\left(p_{i}, r_{i}\right)}{1-\epsilon}=\frac{(1+\epsilon) \Lambda A}{1-\epsilon} .
\end{aligned}
$$

Since $\epsilon$ is arbitrary it follows that $L A \leqq \Lambda A$. But $\Lambda A \leqq L A$; hence we have the following result.

Theorem 3. If $A$ is a plane regular point set, then

$$
h L A=L^{(2)} J_{h}=\Lambda^{(2)} J_{h}=h \Lambda A .
$$

11. The Condition not Necessary. We now give an example of a 
set $A$ which is not regular, but for which $L A=\Lambda A$. This set was constructed by Sierpinski [7], page 184, to show the existence of a set which is not regular.

Let $T_{0}$ be an equilateral triangle of side 1 . In $T_{0}$ form three equilateral triangles of side $1 / 3$, each with a vertex and two sides in common with $T_{0}$. Call the union of these three triangles $T_{1}$. Now proceed with each triangle of $T_{1}$ as was done with $T_{0}$, thus obtaining a set $T_{2}$ consisting of $3^{2}$ triangles each of side $1 / 3^{2}$. In general $T_{n}$ consists of $3^{n}$ equilateral triangles each of side $1 / 3^{n}$. The set $A$ is then the intersection $T_{0} T_{1} T_{2} \cdots$.

Since the projection of $A$ on any side of $T_{0}$ completely fills that side, $L A \geqq 1$ and $\Lambda A \geqq 1$. On the other hand, for each $n$, the point set $T_{n}$ is a covering of $A$ by $3^{n}$ convex point sets each with diameter $1 / 3^{n}$. Thus $L_{1 / 3^{n}} A \leqq 3^{n}\left(1 / 3^{n}\right)=1$, so $L A \leqq 1$. But $\Lambda A \leqq L A$, so we have $L A=\Lambda A=1$.

To see that $A$ is not regular, let $p$ be a point of $A$. Then $p$ belongs to some triangle of $T_{n}$ for every $n$. Also the circle $c\left(p, 1 / 3^{n}\right)$ contains all of one triangle of $T_{n}$ and not more than one point of any other triangle of $T_{n}$. Consequently,

$$
\frac{L A c\left(p, 1 / 3^{n}\right)}{2\left(1 / 3^{n}\right)}=\frac{1 / 3^{n}}{2\left(1 / 3^{n}\right)}=\frac{1}{2} \text {. }
$$

We thus see that the lower density of $A$ is less than or equal to $1 / 2$ at each point of $A$, and $A$ is not regular.

\section{BIBLIOGRAPHY}

1. A. S. Besicovitch, On the fundamental geometrical properties of linearly measurable plane sets of points, Mathematische Annalen, vol. 98 (1927), pp. 422-464.

2. C. Carathéodory, Über das lineare Mass von Punktmengen - eine Verallgemeinerung des Längenbegriffs, Göttingen Nachrichten, 1914, pp. 404-426.

3. W. Gross, Über Flächenmass von Punktmengen, Monatshefte für Mathematik und Physik, vol. 29 (1918), pp. 145-176; and Über das lineare Mass von Punktmengen, same volume, pp. 177-193.

4. Hans Hahn, Theorie der Reellen Funktionen, vol. 1, 1921.

5. J. F. Randolph, Caratheodory measure and a generalization of the GaussGreen lemma, Transactions of this Society, vol. 38 (1935), pp. 531-548.

6. S. Saks, Remarque sur la mesure linéaire des ensembles plans, Fundamenta Mathematicae, vol. 9 (1927), pp. 16-24.

7. W. Sierpinski, Sur la densité linéaire des ensembles plans, Fundamenta Mathematicae, vol. 9 (1927), pp. 172-185.

Cornell University 